\title{
Novel Wireless Thermal Convection Angular Accelerometer Integrated with Radio Frequency Identification Tag
}

\author{
Jium-Ming Lin*, Cheng-Hung Lin ${ }^{1}$ and Hung-Han $\mathrm{Lu}^{2}$ \\ Department of Communication Engineering, Chung-Hua University, Hsin-Chu 30012, Taiwan \\ ${ }^{1}$ Engineering Science, Chung-Hua University, Hsin-Chu 30012, Taiwan \\ ${ }^{2}$ Department of Electrical Engineering, Chung-Hua University, Hsin-Chu 30012, Taiwan
}

(Received November 5, 2012; accepted February 4, 2013)

Key words: angular accelerometer, RFID tag, flexible substrate, hemispherical chamber, xenon gas

Five novel ideas are proposed to integrate a radio frequency identification (RFID) tag with a thermal convection angular accelerometer on a flexible substrate. The first idea is that this device has no movable parts; therefore, it is very reliable. The second idea is that the thermal conductivity of flexible substrates, such as plastic or polyimide, is much lower than that of silicon, and thus, it can save more power and is very useful for mobile operations. The third new idea is to apply xenon gas to the chamber to conduct heat instead of $\mathrm{CO}_{2}$ conventionally used, which can oxidize the heater and thermal sensors, while the inert gas xenon cannot. The fourth new idea is the use of a hemispherical chamber; it is more streamlined with less drag to yield quicker responses. The fifth new idea is to integrate an angular accelerometer with the RFID tag on the same flexible substrate; thus, the device becomes a more useful wireless sensor. Compared with a device fabricated using a rectangular chamber filled with $\mathrm{CO}_{2}$, we noted that the linearity, sensitivity, and response time of step-input angular acceleration are better for the proposed device. The sensitivity is $71.4^{\circ} \mathrm{C} /\left(\mathrm{rad} / \mathrm{s}^{2}\right)$ and the response time is $60 \mu \mathrm{s}$.

\section{Introduction}

Conventional accelerometers are fabricated on silicon wafers, ${ }^{(1-17)}$ and some of them are fabricated using thermal bubble technologies and a chamber is filled with air, $\mathrm{CO}_{2}$, liquid, or others. As shown in Fig. 1, five novel ideas are proposed in this paper to integrate an RFID tag with thermal bubble angular accelerometers on a flexible substrate. The first idea is that the new device has no movable parts; therefore, it is very reliable. The second idea is to form the device on a flexible substrate, such as plastic or polyimide; the thermal conductivity of plastic $[0.06-0.0017 \mathrm{~W} /(\mathrm{cm} \cdot \mathrm{K})]$ is about twenty-fifth that of

${ }^{*}$ Corresponding author: e-mail: jmlin@chu.edu.tw 
silicon $[1.48 \mathrm{~W} /(\mathrm{cm} \cdot \mathrm{K})]$; thus, we can save more power by preventing leakage through the substrate. The third idea is to fill the chamber with xenon gas for heat conduction instead of $\mathrm{CO}_{2}$ previously used, which can oxidize the heater and thermal sensors, ${ }^{(1-18)}$ while the xenon gas cannot. Therefore, we can increase heater reliability and life cycle. Moreover, the molecular weight $(131.29 \mathrm{~g} / \mathrm{mol})$ of Xe is three times that of $\mathrm{CO}_{2}(44.01$ $\mathrm{g} / \mathrm{mol}$ ); the inertia of $\mathrm{Xe}$ is larger and can yield quicker responses. The fourth idea is to use a hemispherical chamber; it is more streamlined with less drag to yield quicker responses. Thus, both the sensitivity and bandwidth of packages fabricated using our proposed system are larger than those of the conventional packages fabricated using a rectangular chamber. ${ }^{(18)}$ For the outer case, the rectangular type can still be used for easy labeling of the name and series number. The fifth idea is the most powerful one, that is, to integrate an RFID tag with an angular accelerometer on the same substrate; thus, the device becomes a more useful wireless sensor, which can be applied to many fields. The heater is made of chromium (Cr) and nickel (Ni), as shown in Fig. 2, and its operating temperature is $127{ }^{\circ} \mathrm{C}(400 \mathrm{~K})$, at which polyimide does not melt. Compared with a device fabricated using a rectangular chamber filled with $\mathrm{CO}_{2}$, we can see that the linearity, sensitivity, and response time of step-input angular acceleration are better for the hemispherical chamber filled with Xe gas. The sensitivity is $71.4{ }^{\circ} \mathrm{C} /\left(\mathrm{rad} / \mathrm{s}^{2}\right)$ and the response time is $60 \mu \mathrm{s}$. The paper organization is as follows: the first section is the introduction. The next section concerns the fabrication and packaging steps. The third section is simulation and discussion. Finally, a summary is given.

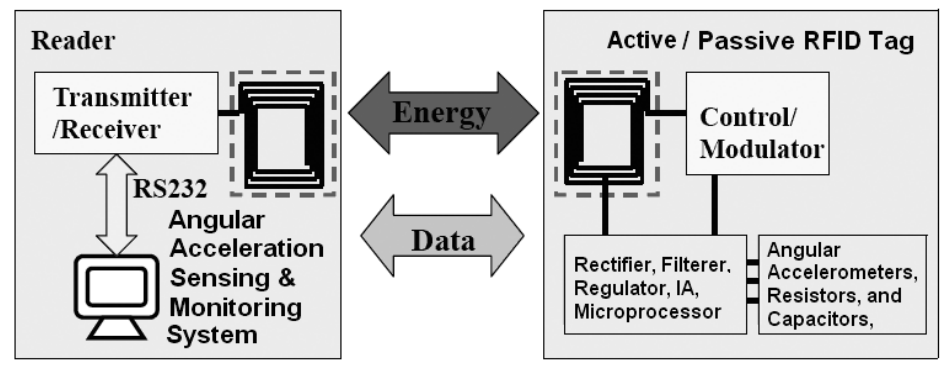

Fig. 1. Block diagram of proposed RFID-based angular accelerometer.

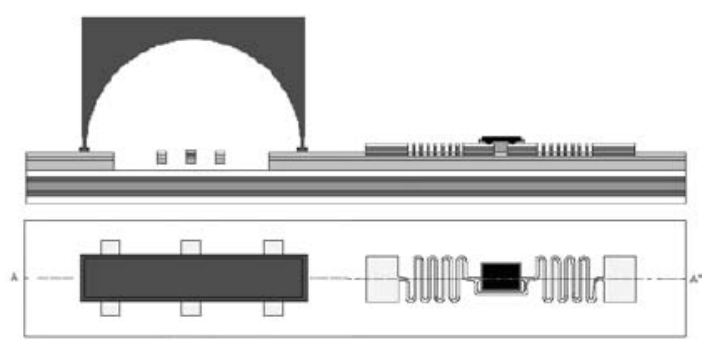

Fig. 2. Side and top views of device. 


\section{Fabrication and Packaging Steps}

Step 1: Deposit layers of $\mathrm{SiO}_{2}$ and a photoresist (PR) on both surfaces for thermal, electrical, and humidity isolation. Use mask \#1 and photolithography and etching processes (PAEP) to form a cavity on the surface. Deposit $\mathrm{SiO}_{2}$ as the sacrificial layer. Use mask \#1 and PAEP, only retain $\mathrm{SiO}_{2}$ on the cavity, as shown in Fig. 2.

Step 2: Evaporate a layer of alumina oxide, use mask \#2 and PAEP to retain the alumina oxide layers to support the heater and temperature sensors. Deposit p-type (such as boron) amorphous silicon, then use a Nd-YAG laser to anneal it to form a poly$\mathrm{Si}$ thermistor without melting polyimide. Use mask \#3 and PAEP to retain two layers of poly-Si thermistor. As shown in Fig. 2, deposit $\mathrm{Cr}$ and Ni to form the heater, and the supporting layers of the RFID antenna, and the conductors.

Step 3: Use mask \#2 and PAEP to release the heater and thermistors. Apply screen printing to place a plastic material around the angular accelerometer as a dam bar, put a square flat cap package ${ }^{(18)}$ with a hemispherical chamber on the dam bar, then cure the device by filling the chamber with Xe. Flip-chip bond the chip with bumps to the RFID antenna feed terminals, and then form the underfill to increase chip adherence; the result is as shown in Fig. 2.

\section{Simulation and Discussion}

We use the ESI-CFD+ software package for simulation. Firstly, the device geometric dimensions are shown in Fig. $3(H=19.7 \mathrm{~mm}, S=2 \mathrm{~mm}, W 1=2 \mathrm{~mm}$, and $W 2=0.3$ $\mathrm{mm}$ ). The temperatures of the package boundaries and heater are set at 300 and 400 $\mathrm{K}$, respectively. As in Fig. 3, the thermal sensor can be put at any of the three points to be trade-off by simulation. Firstly, using the square chamber filled with Xe gas, the sensitivities (thermal sensors temperature difference vs angular acceleration) of the thermal sensors at points 1, 2, and 3 are determined and shown in Fig. 4. Note that the sensitivity is better when the thermal sensors are placed at point 2 , although the linearity is not good. Therefore, we put the thermal sensors at this place for the following analysis. The step-input angular acceleration responses of total enthalpy are also listed in Table 1. For comparison, the results in the case of filling the square chamber with $\mathrm{CO}_{2}$
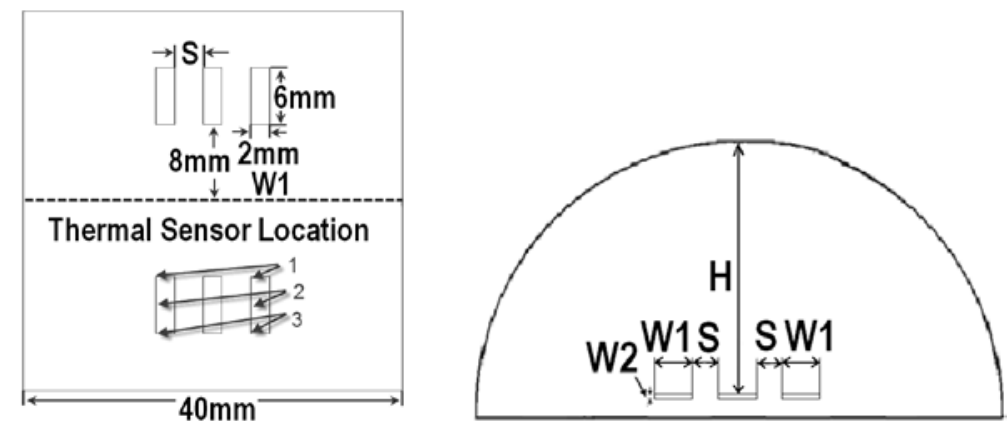

Fig. 3. Geometric dimensions of square and hemispherical chambers. 


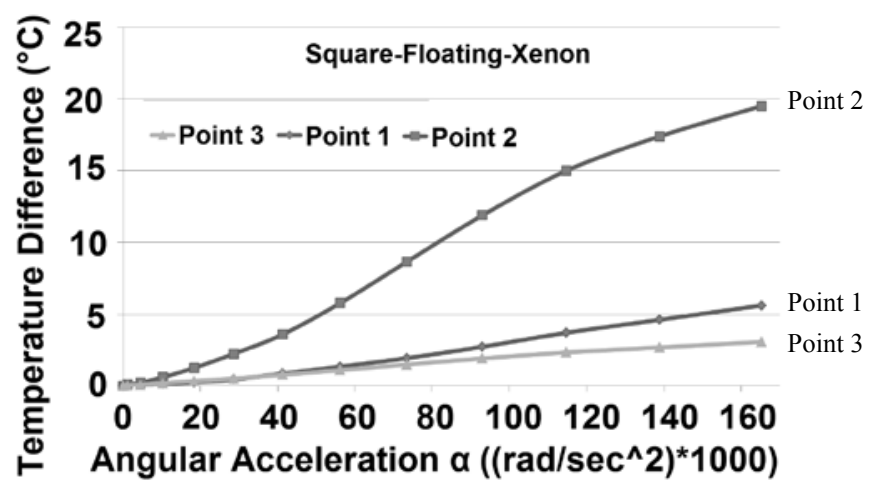

Fig. 4. Sensitivities in the case of using square chamber and Xe gas.

Table 1

Response times of step-input angular acceleration.

\begin{tabular}{lccccc}
\hline \multirow{2}{*}{ Items } & \multicolumn{4}{c}{ Chambers } \\
& \multicolumn{1}{c}{ Hemispherical $(\mu \mathrm{s})$} & \multicolumn{2}{c}{ Square $(\mu \mathrm{s})$} \\
\cline { 2 - 3 } \cline { 5 - 6 } & $\mathrm{Xe}$ & $\mathrm{CO}_{2}$ & & $\mathrm{Xe}$ & $\mathrm{CO}_{2}$ \\
\hline Static pressure & 60 & 75 & & 70 & 106 \\
Total enthalpy & 40 & 46 & & 47 & 67 \\
\hline
\end{tabular}

are also shown in Fig. 5. Note that the sensitivity is also better when the thermal sensors are placed at point 2. From Figs. 4 and 5 and Table 1, we note that both the sensitivity and response time are better for the chamber filled with Xe gas.

In the case of using the hemispherical chamber filled with Xe, the sensitivities of the thermal sensors at points 1, 2, and 3 are shown in Fig. 6 . Note that the sensitivity (71.4 ${ }^{\circ} \mathrm{C} /\left(\mathrm{rad} / \mathrm{s}^{2}\right)$ and linearity are also better when the thermal sensors are placed at point 2 . Therefore, we put the thermal sensors at this place for the following analysis. The stepinput angular acceleration responses of Xe gas total enthalpy are also listed in Table 1.

For comparison, the results in the case of filling the hemispherical chamber with $\mathrm{CO}_{2}$ are also shown in Fig. 7. Note that the sensitivity is also better for the thermal sensors placed at point 2. Moreover, the sensitivity is also better for the chamber filled with Xe. The detailed response times of step-input angular acceleration in the case of using Xe and $\mathrm{CO}_{2}$ gases are also listed in Table 1. From Figs. 6 and 7, we can see that the linearity and sensitivity are better for the hemispherical chamber filled with Xe gas. From Table 1, we can see that the response times of step-input angular acceleration for the hemispherical chamber filled with Xe gas are also better. Moreover, the sensitivity is $71.4{ }^{\circ} \mathrm{C} /\left(\mathrm{rad} / \mathrm{s}^{2}\right)$ and the response time is $60 \mu \mathrm{s}$. 


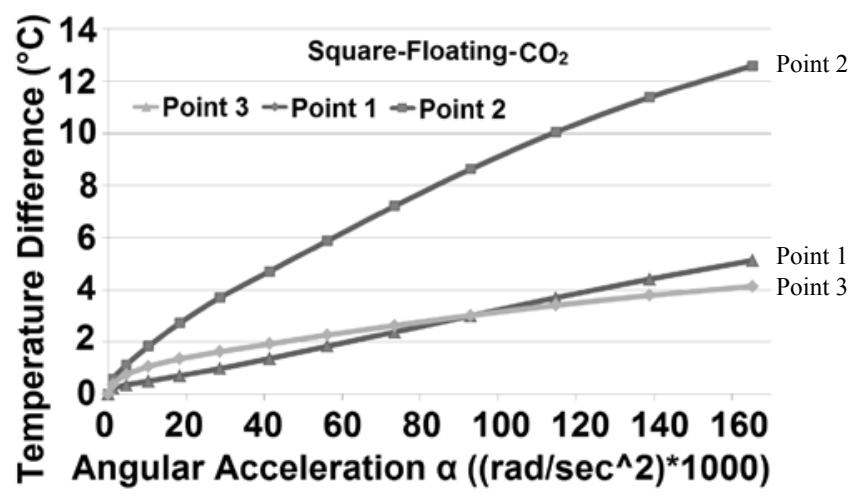

Fig. 5. Sensitivities in the case of using square chamber and $\mathrm{CO}_{2}$ gas.

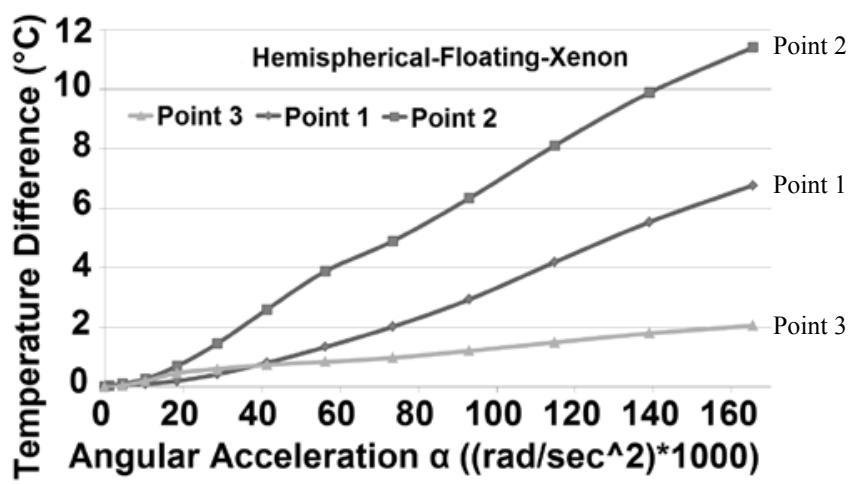

Fig. 6. Sensitivities in the case of using hemispherical chamber with Xe gas.

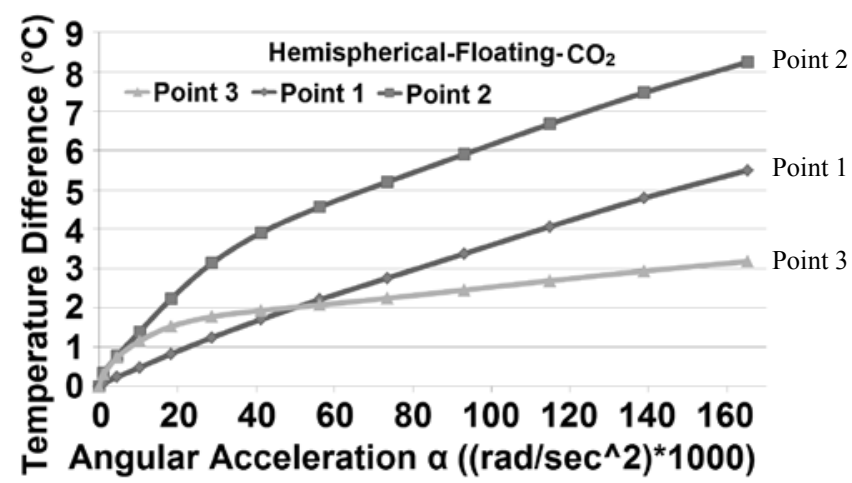

Fig. 7. Sensitivities in the case of using hemispherical chamber and $\mathrm{CO}_{2}$ gas. 


\section{Summary}

The contributions of this study are summarized as follows: It is a novel idea to fabricate both the heater and temperature sensors of a thermal convection-type angular accelerometer on a flexible substrate, which has not been proposed. Thus, one can integrate the RFID tag with the angular accelerometer on the plastic substrate to fabricate a more powerful wireless angular acceleration sensor. The use of a flexible substrate is a new idea; its thermal isolation capability is better than that of silicon with the plastic material, thus the cost of power dissipation during long-term operation is lower for the new design. Moreover, the inert Xe gas is more reliable without the oxidation and aging effects. We can see that the linearity, sensitivity, and response time of step-input angular acceleration are better for the hemispherical chamber filled with Xe gas. The sensitivity is $71.4{ }^{\circ} \mathrm{C} /\left(\mathrm{rad} / \mathrm{s}^{2}\right)$ and the response time is $60 \mu \mathrm{s}$.

\section{References}

1 L. Lin, R. T. Howe and A. P. Pisano: J. Microelectromech. Syst. 7 (1998) 286.

2 A. Khalkhali, M. Farajpoor and H. Safikhani: Trans. Can. Soc. Mech. Eng. 35 (2011) 63.

3 C. K. Varghese and K. Shankar: Int. J. Eng. Technol. Innov. 1 (2011) 65.

4 D. Dubé and P. Cardou: Trans. Can. Soc. Mech. Eng. 35 (2011) 251.

5 C. Y. Lin, J. F. Jiang, Y. J. Chen, H. T. Wei and H. A. Chen: Trans. Can. Soc. Mech. Eng. 36 (2012) 97.

6 Y. Zhao, A. Leung, M. E. Rebeschini, G. P. Pucci, Dribinsky and B. A. Y. Cai: U.S. Patent No. 7305881 B2 (2007).

7 Y. Zhao, A. P. Brokaw, M. E. Rebeschini, A. M. Leung, G. P. Pucci and A. Dribinsky: U.S. Patent No. 6795752 B1 (2004).

8 K. M. Liao, R. Chen and B. C. S. Chou: Sens. Actuators, A 282 (2006) 130.

9 U. A. Dauderstadt, P. M. Sarro and S. Middelhoek: Solid State Sens. Actuators 2 (1997) 1209.

10 R. Dao, D. E. Morgan, H. H. Kries and D. M. Bachelder: U.S. Patent No. 5581034 (1996).

11 V. Milanovic, E. Bowen, N. Tea, J. S. Suehle, B. Payne, M. E. Zaghloul and M. Gaitan: Proc. Int. Mech. Eng. Conf. Exposition, MEMS Symposia (MEMS Symposia and Anaheim, California, 1998) p. 627.

12 X. B. Luo, Z. X Li, Z. Y. Guo and Y. J. Yang: J. Micromech. Microeng. 11 (2001) 504.

13 L. Lin, A. P. Pisano and V. P. Carey: Microsyst. Technol. 1 (1994) 51.

14 J. H. Tsai and L. Lin: Sens. Actuators, A 97 (2002) 1.

15 J. Dido, P. Loisel and A. Renault: U.S. Patent No. 7426862 B2 (2008).

16 J. Courteaud, N. Crespy, P. Combette, B. Sorli and A. Giani: Sens. Actuators, A 147 (2008) 75.

17 U. A. Dauderstädt, P. H. S. de Vries, R. Hiratsuka, J. G. Korvink, P. M. Sarro, H. Baltes and S. Middelhoek: Sens. Actuators, A 55 (1996) 3.

18 T. R. Hsu: In MEMS \& Microsystems: Design and Manufacture (McGraw-Hill Companies, Inc., Boston, 2002) p. 201. 